\title{
Reversión del bypass gástrico: Indicaciones y resultados quirúrgicos
}

\author{
Nicolás Besser-Pimentel ${ }^{1}$, Pedro Pablo Soto-Gajardo ${ }^{1}$, Pablo Achurra-Tirado ${ }^{1}$, \\ Nicolás Quezada-Sanhueza ${ }^{1}$, Alejandro Raddatz-Echavarría ${ }^{1}$, Fernando Crovari-Eulufi ${ }^{1}$, \\ Gustavo Pérez-Blanco ${ }^{1}$, Ricardo Mejía-Martínez ${ }^{1}$, Mauricio Gabrielli-Nervi', \\ Julieta Klaassen-Lobos ${ }^{1}$, Sergio Guzmán-Bondiek ${ }^{1}$ y Rodrigo Muñoz-Claro ${ }^{1}$
}

\section{Gastric bypass reversal: Surgical indications and results}

Introduction: Due to its efficacy in the treatment of morbid obesity, roux-en-y gastric bypass (RYGB) continues to be a frequently performed intervention. However, a small group of patients may develop nutritional and metabolic complications that cannot be controlled with optimal medical treatment. In these cases, it may be necessary to reestablish the continuity of the gastrointestinal tract by reversing the RYGB (R-RYGB). Aim: To present the indications and results obtained in a series of patients who underwent to R-RYGB. Materials and Method: Identification and retrospective evaluation of all patients who underwent consecutive R-RYGB in our institution. Demographic and anthropometric characteristics of the original surgery and at the time of the reversal were recorded. Complications were classified according to Clavien-Dindo classification. Results: Seven patients were identified in whom an R-RYGB was performed. In 2 cases the reversal was due to short bowel syndrome, in 3 cases due to severe hypoglycemia refractory to medical treatment and in 2 cases due to chronic diarrhea. The median age at the time of the reversal was 55 years. The median time from primary surgery to reversal was 77 months. The median hospital stay was 6 days. There were no Clavien-Dindo complications $\geq$ III. The R-RYGB was able to reverse nutritional and metabolic complications in all cases. Conclusion: Restoring the continuity of the gastrointestinal tract allows control of nutritional and metabolic complications.

Key words: obesity; gastric bypass; complications; reversal.

\section{Resumen}

Introducción: Debido a su eficacia en el tratamiento de la obesidad mórbida, el bypass gástrico (BPG) sigue siendo una intervención realizada frecuentemente. Sin embargo, un grupo reducido de pacientes puede desarrollar complicaciones nutricionales y metabólicas que no logran controlarse con un tratamiento médico óptimo. En estos casos, puede ser necesario reestablecer la continuidad del tracto gastrointestinal por medio de la reversión del BPG (R-BPG). Objetivo: Presentar las indicaciones y resultados obtenidos en una serie de pacientes sometidos a una R-BPG. Materiales y Método: Identificación y evaluación retrospectiva de todos los pacientes sometidos a una R-BPG en nuestra institución de manera consecutiva. Se registraron las características demográficas y antropométricas de la cirugía original y al momento de la reversión. Las complicaciones se registraron de acuerdo con la clasificación de Clavien-Dindo. Resultados: Se identificaron 7 pacientes en los cuales se realizó una R-BPG. En 2 casos la reversión fue por síndrome de intestino corto, en 3 casos por hipoglicemias severas refractarias a manejo médico y en 2 casos por diarrea crónica. La mediana de edad al momento de la reversión fue de 55 años. La mediana de tiempo desde la cirugía original hasta el momento de la reversión fue de 77 meses. La mediana de estadía hospitalaria fue de 6 días. No hubo complicaciones Clavien-Dindo $\geq$ III. La R-BPG logró revertir en todos los casos las complicaciones nutricionales y metabólicas. Conclusión: La restauración de la continuidad del tracto gastrointestinal permite el control de las complicaciones nutricionales y metabólicas. Palabras clave: obesidad; bypass gástrico; complicaciones; reversión.
'Departamento Cirugía Digestiva Pontificia

Universidad Católica de Chile. Santiago, Chile.

Recibido 2020-06-20 y aceptado 2020-09-06

Correspondencia a: Dr. Nicolás Besser-Pimentel rmunozc@med.puc.cl 


\section{Introducción}

El tratamiento quirúrgico de la obesidad sigue siendo la intervención más eficaz para lograr una baja de peso significativa y mantenida en el tiempo ${ }^{1,2}$. Adicionalmente a la pérdida de peso experimentada por los pacientes después de la cirugía, muchas de las enfermedades asociadas o exacerbadas por la obesidad logran mejorar significativamente, llegando algunas incluso a remitir ${ }^{3,4}$. Debido a sus múltiples beneficios y seguridad, en la actualidad el bypass gástrico (BPG) laparoscópico continúa siendo un procedimiento realizado frecuentemente ${ }^{5,6}$. Sin embargo, después de esta cirugía un número reducido de pacientes puede desarrollar complicaciones infrecuentes, pudiendo llegar a requerir en ocasiones la reversión del bypass (R-BPG) para poder controlar los efectos de estas complicaciones por medio de la restauración la continuidad del tracto gastrointestinal. La R-BPG se realiza esporádicamente, siendo por primera vez reportada por Himpens el año $2006^{7}$. A partir de esa fecha se pueden encontrar en la literatura múltiples publicaciones, correspondientes en su mayoría a reportes de caso o series que incluyen un número reducido de casos.

Las causas más comunes para indicar una RBPG son la desnutrición secundaria a un síndrome de intestino corto, la neuroglucopenia secundaria a hipoglicemia, diarrea crónica, úlceras anastomóticas recurrentes, síndrome de dumping, complicaciones anatómicas (pseudoobstrucción intestinal) y síntomas funcionales crónicos (náuseas, vómitos y dolor abdominal), entre otros ${ }^{8-11}$.
En nuestra institución, el primer BPG se realizó el año 1992 por vía abierta, para luego el año 2001 incorporar el abordaje laparoscópico ${ }^{12}$. En nuestra práctica, la R-BPG es un procedimiento infrecuente. Por esta razón, el objetivo de este trabajo es presentar nuestra experiencia y resultados con la R-BPG.

\section{Materiales y Método}

Se identificaron todos los pacientes que fueron sometidos de manera consecutiva a una R-BPG en el Hospital Clínico de la Pontificia Universidad Católica de Chile. A partir de nuestros registros clínicos y base de datos se obtuvieron las características demográficas, indicación de BPG, tiempo entre el BPG y la reversión, edad al momento de la reversión, técnica empleada y complicaciones de acuerdo con la clasificación Clavien-Dindo ${ }^{13}$. Las variables continuas se presentan con medianas y rangos.

\section{Técnica de reversión del BPG}

Se utilizaron dos técnicas para la R-BPG (Figura 1A), una con anastomosis gastro-gástrica y otra utilizando un puente de yeyuno. La técnica con anastomosis gastro-gástrica (Figura 1B) se efectuó de la siguiente manera: primero se realiza la desconexión de la gastroyeyuno anastomosis mediante su sección con una engrapadora lineal. Así, se libera el extremo proximal del asa alimentaria, la cual quedará libre para su posterior anastomosis con el asa biliopancreática. Luego se realiza anastomosis gastro-gástrica entre la cara lateral de la bolsa gástrica y el estómago remanente de forma mecánica.
Figura 1. Técnicas para la R-BPG. A) BPG estándar. B) Reversión mediante anastomosis gastro-gástrica. C) Reversión mediante interposición de yeyuno.
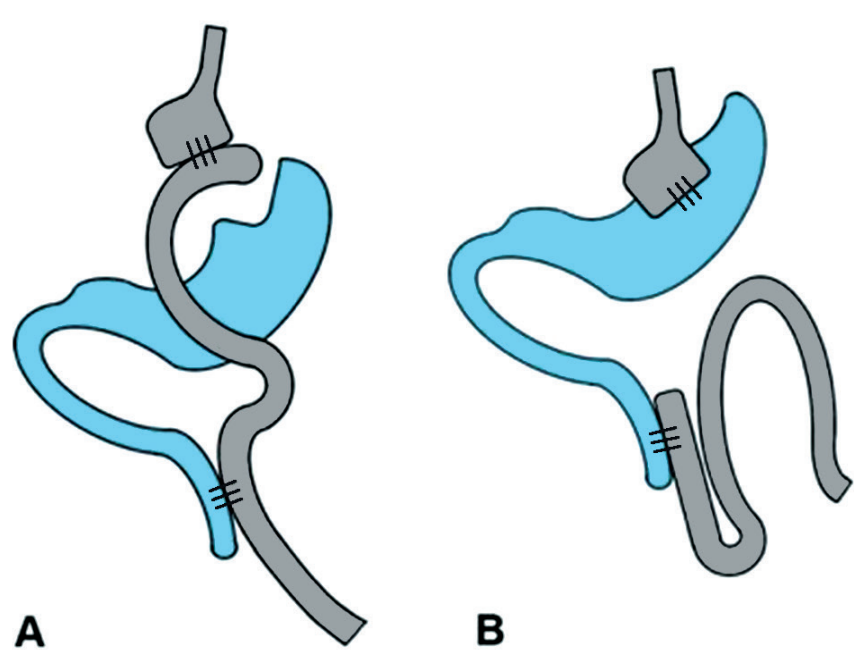

B

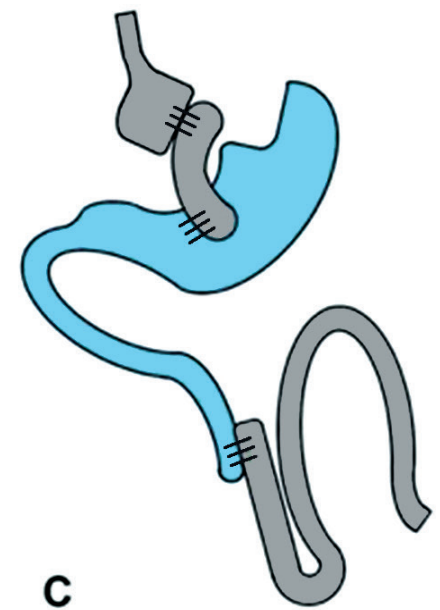


Se comprueba la permeabilidad de esta anastomosis con sonda de 50 Fr. A continuación, se identifica la entero-entero anastomosis y el extremo distal del asa alimentaria. Posteriormente, se confecciona la anastomosis latero-lateral mecánica entre el extremo proximal del asa alimentaria y el extremo distal del asa biliopancreática, reconstituyendo la continuidad del tracto gastrointestinal. Finalmente se cierra la brecha mesentérica con sutura irreabsorbible.

Como variante técnica utilizamos un puente de yeyuno entre la bolsa gástrica y el antro gástrico (Figura 1C). Seccionamos con engrapadora lineal el asa alimentaria que asciende justo anterior al antro, quedando así disponible un puente de yeyuno de aproximadamente $15 \mathrm{~cm}$ desde la bolsa gástrica hasta el antro del estómago excluido. Este puente de yeyuno se conecta al antro gástrico por medio de una anastomosis manual. Posteriormente se identifica la entero-entero anastomosis, se secciona el extremo del asa biliopancreática y se realiza una anastomosis mecánica entre este extremo y el extremo del asa alimentaria anteriormente seccionada, restaurando la continuidad del tracto gastrointestinal.

\section{Resultados}

Entre enero de 2013 y mayo de 2020 se realizaron un total de 2.826 cirugías bariátricas, de las cuales un $55 \%(\mathrm{n}=1.554)$ correspondieron a BPG, un $45 \%(\mathrm{n}=1.272)$ a gastrectomía en manga $(\mathrm{GM})$ y un $3,7 \%(n=107)$ conversión de GM a BPG. En este período 7 pacientes $(0,25 \%)$ fueron sometidos a una R-BPG, de los cuales 4 fueron mujeres. Las características de los pacientes se resumen en la Tabla 1. La mediana de edad y del tiempo transcurrido desde la cirugía primaria hasta la reversión fue de 55 años (46-63) y de 77 meses (10-132), respectivamente. La mediana de duración de la cirugía fue de 150 minutos (120-420) y de la estadía hospitalaria fue de 6 días (3-49). A continuación se detallan los casos de forma individual y consecutiva.

\section{Síndrome de intestino corto}

\section{Caso 1}

Hombre de 62 años, con un índice de masa corporal (IMC) de $40,7 \mathrm{~kg} / \mathrm{m}^{2}$ asociado a hipertensión arterial (HTA), diabetes mellitus (DM) no insulinorequirente y esteatosis hepática. Se realizó un $\mathrm{BPG}$ laparoscópico en nuestro centro el año 2011. A los 21 meses después de la cirugía consultó en el servicio de urgencia de otro centro hospitalario, en el cual se identificó una hernia interna, siendo sometido a una resección intestinal extensa por vía abierta. Evolucionó con hipoalbuminemia y síndrome de intestino corto, requiriendo hospitalizaciones prolongadas y nutrición parenteral. Siete meses después de la resección intestinal y debido a la mala respuesta al manejo médico se decidió R-BPG. La técnica utilizada fue la reversión con anastomosis gastro-gástrica (Figura 1B) por vía abierta. Al momento de la reversión, el asa biliar, alimentaria y común tenían una longitud de 60,150 y $500 \mathrm{~cm}$ respectivamente. El paciente evolucionó con infección de la herida operatoria, la cual se manejó con curaciones y tratamiento antibiótico. Fue dado de alta 14 días después de la cirugía.

Tabla 1. Características demográficas, indicación y resultados perioperatorios de pacientes sometidos a reversión del bypass gástrico (R-BPG)

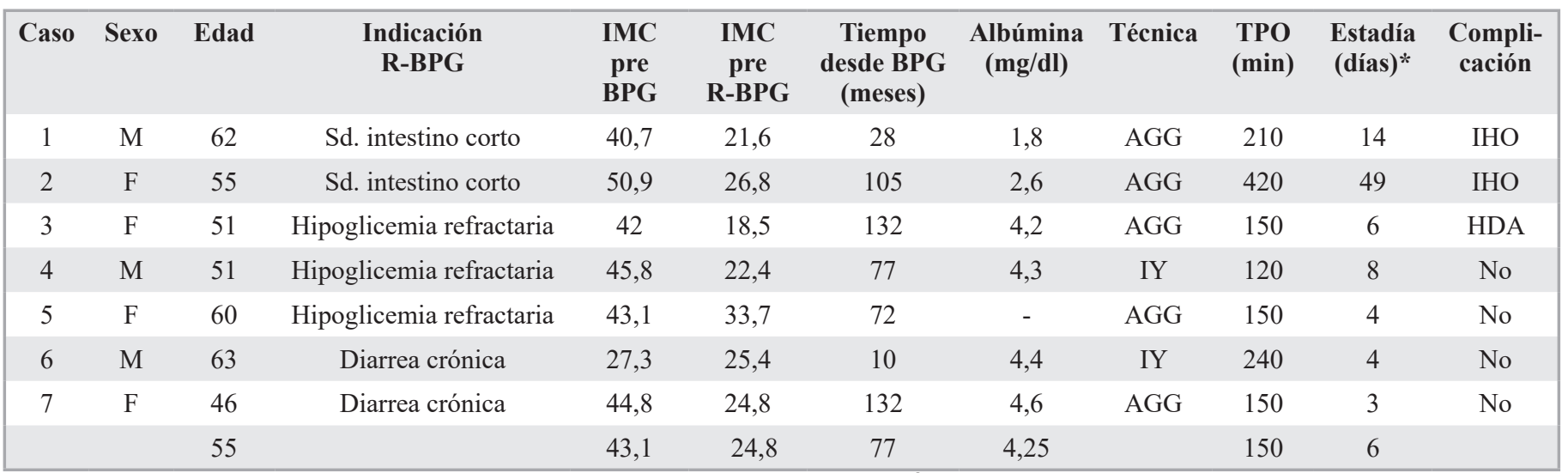

*Posterior a realización de la R-BPG. Mediana (Me). Masculino (M). Femenino (F). Índice de masa corporal (IMC). Anastomosis gastro-gástrica (AGG). Interposición de yeyuno (IY). Tiempo operatorio (TO). Infección de herida operatoria (IHO). Hemorragia digestiva alta (HDA) 


\section{Caso 2}

Mujer de 55 años, IMC de $50,9 \mathrm{~kg} / \mathrm{m}^{2}$ asociado a HTA y DM no insulino-requirente. Se realizó un BPG laparoscópico en otro centro el año 2007. Seis años después fue intervenida por una obstrucción intestinal por bridas, siendo sometida a una resección intestinal extensa más confección de ileostomía. Luego de 21 meses de la última cirugía se realizó la reconstitución de tránsito intestinal y evolucionó con hipoalbuminemia severa por síndrome de intestino corto, la cual fue refractaria al manejo médico, requiriendo múltiples hospitalizaciones y apoyo con nutrición parenteral. Debido a su compromiso nutricional se decidió R-BPG.

Se efectuó la R-BPG mediante la técnica gastrogástrica (Figura 1B) por vía abierta 11 meses después del cierre de la ileostomía. Evolucionó con infección de la herida operatoria por un microorganismo multirresistente, requiriendo tratamiento antibiótico prolongado y uso de VAC (Vacuum Assisted Closure). Tras completar 49 días después de la cirugía, la paciente fue dada de alta.

\section{Hipoglicemias refractarias}

\section{Caso 3}

Mujer de 51 años, IMC de $42 \mathrm{~kg} / \mathrm{m}^{2}$ asociado a esteatosis hepática. Sometida a BPG laparoscópico en nuestra institución el año 2005. Consultó 10 años después de la cirugía por síntomas compatibles con hipoglicemias objetivadas hasta $17 \mathrm{mg} / \mathrm{dl}$. Se realizó manejo médico el cual tuvo mala respuesta, manteniendo episodios de hipoglicemias severas sintomáticas y hospitalizaciones en diferentes oportunidades.

Se decidió realizar la R-BPG después de 1 año del inicio de síntomas por vía abierta usando la técnica gastro-gástrica (Figura 1B). En el posoperatorio evolucionó con una hemorragia digestiva alta secundaria a sangrado de la anastomosis gastrogástrica, que se logró manejar exitosamente por vía endoscópica con clips hemostáticos. La paciente fue dada de alta en buenas condiciones 6 días después de su cirugía.

\section{Caso 4}

Hombre de 51 años, IMC de $45,8 \mathrm{~kg} / \mathrm{m}^{2}$ asociado a resistencia a la insulina. Se realizó un BPG laparoscópico en otro centro el año 2010. Cinco años post-BPG consultó por hipoglicemias severas objetivadas hasta $27 \mathrm{mg} / \mathrm{dl}$. Se inició manejo médico, que no logró revertir los episodios de hipoglicemia sintomática.
Se efectuó R-BPG 17 meses después del inicio de síntomas por laparoscopía, mediante la técnica con puente de yeyuno (Figura 1C). Evolucionó de forma favorable, sin complicaciones, siendo dado de alta al $8^{\circ}$ día posoperatorio.

\section{Caso 5}

Mujer de 60 años, IMC de 43,1 $\mathrm{kg} / \mathrm{m}^{2}$ asociado a HTA, sometida a BPG en otro centro el año 2013. Consultó luego de 6 años por síntomas de dumping e hipoglicemias severas, recurrentes y refractarias a manejo médico.

La R-BPG se realizó 72 meses luego del inicio de síntomas por laparoscopia, usando la técnica gastrogástrica (Figura 1B). Evolucionó sin complicaciones y fue dada de alta al $4^{\circ}$ día posterior a la cirugía.

\section{Diarrea crónica}

\section{Caso 6}

Hombre de 63 años, IMC de $33 \mathrm{~kg} / \mathrm{m}^{2}$ asociado a dislipidemia, resistencia a la insulina y síndrome de apnea obstructiva del sueño. El año 2014 fue sometido a GM y, posteriormente, a una cirugía revisional (con un IMC de $27,3 \mathrm{~kg} / \mathrm{m}^{2}$ ) con conversión a BPG y hernioplastía hiatal por reflujo gastroesofágico en otro centro el año 2018. Consultó en nuestro centro por síndrome diarreico persistente desde la conversión a BPG. Se realizó estudio con biopsias de colon, las cuales resultaron normales. La determinación de la IgA y anticuerpos antiendomisio fue normal. Se descartó sobrecrecimiento bacteriano con test de lactulosa/hidrógeno espirado, fructosa y elastasa fecal normal. Persistió con diarrea a pesar de manejo médico con loperamida, colestiramina y enzimas pancreáticas. Evaluado en equipo multidisciplinario, se indicó R-BPG.

Se realizó la R-BPG por laparoscopía mediante la técnica con puente de yeyuno (Figura 1C) luego de 10 meses de inicio de los síntomas. Al momento de la reversión, el asa biliar, alimentaria y común tenían una longitud de 60, 150 y $500 \mathrm{cms}$ respectivamente. Evolucionó sin complicaciones y fue dado de alta al $4^{\circ}$ día posterior a la cirugía.

\section{Caso 7}

Mujer de 46 años, IMC de 44,8 kg/m² sometida a BPG el año 2009 en otro centro. Evolucionó con síndrome diarreico persistente. Consultó en nuestro centro y, posterior a evaluación por nuestro equipo multidisciplinario y considerando deterioro de calidad de vida y refractariedad al tratamiento médico instaurado, se decidió R-BPG. Esta se realizó por 
laparoscopía 132 meses después de la cirugía primaria usando la técnica con anastomosis gastro-gástrica (Figura 1B). En el intraoperatorio se constató el asa biliar, alimentaria y común de 350, 200 y $250 \mathrm{~cm}$. De forma concomitante, se realizó una hernioplastía hiatal. Evolucionó de manera satisfactoria, siendo dada de alta el $3^{\circ}$ día posoperatorio.

En todos los casos presentados en esta serie, la R-BPG logró revertir las complicaciones y normalizar los parámetros metabólicos y nutricionales. La mediana de seguimiento e IMC fue de 18 meses y $27,8 \mathrm{~kg} / \mathrm{m}^{2}$ respectivamente (Tabla 2 ).

\section{Discusión}

El BPG tiene beneficios ampliamente demostrados en la literatura ${ }^{1,4,14}$. Sin embargo, existe un grupo menor de pacientes que desarrollan complicaciones que pueden llevar a realizar una R-BPG como último recurso terapéutico.

Las indicaciones más frecuentes para realizar una R-BPG reportadas en la literatura son úlceras marginales recurrentes ${ }^{9,11,15-17}$, hipoalbuminemia severa $^{9,10,15,17-19}$ e hipoglicemias ${ }^{10,16,18,20-23}$ (Tabla 3). Otras causas son el dolor abdominal crónico ${ }^{10,15}$, dumping persistente ${ }^{22}$, fístulas gastro-gástricas ${ }^{16} \mathrm{e}$ incluso intususcepciones recurrentes ${ }^{24}$. En nuestra serie, las causas más frecuentes fueron la hipoglicemia, la desnutrición secundaria a intestino corto y la diarrea crónica, concordando con las causas más reportadas en la literatura.

Con respecto a la hipoglicemia hiperinsulinémica posprandial, su prevalencia exacta post-BPG es desconocida, pero se estima que puede ocurrir entre un $10 \%-75 \%$, variando según el método y criterio diagnóstico usado para su definición ${ }^{25-27}$. En nuestros pacientes la R-BPG logró revertir la hipoglicemia de forma exitosa al igual que lo reportado en la literatura $^{19,28,29}$.

Dos pacientes presentaron obstrucción intestinal por hernia interna y por bridas respectivamente. Ambos requirieron resección intestinal masiva, evolucionando con un síndrome de intestino corto. La obstrucción intestinal por hernia interna postBPG es una complicación potencialmente grave pero prevenible con un diagnóstico oportuno, con una incidencia reportada en la literatura que oscila en un rango de $1 \%-11 \%{ }^{30,31}$. El cierre sistemático de la brecha mesentérica ha demostrado reducir su incidencia en un $44 \% \%^{32}$. En nuestro centro, el cierre de esta brecha y el cierre del espacio de Petersen se hacen de forma rutinaria. Con respecto al diagnóstico oportuno, la incorporación de la tomografía
Tabla 2. Seguimiento de pacientes sometidos a R-BPG

\begin{tabular}{|ccccc|}
\hline Caso & $\begin{array}{c}\text { Fecha cirugía } \\
\text { (mes/año) }\end{array}$ & $\begin{array}{c}\text { Seguimiento } \\
\text { (meses) }\end{array}$ & IMC & $\begin{array}{c}\text { Resolución } \\
\text { de síntomas }\end{array}$ \\
1 & $12 / 2013$ & 82 & 27,8 & Sí \\
2 & $09 / 2015$ & 60 & 33,3 & Sí \\
\hline 3 & $08 / 2016$ & 13 & 21,1 & Sí \\
4 & $11 / 2016$ & 47 & 28,4 & Sí \\
\hline 5 & $03 / 2019$ & 18 & 33,8 & Sí \\
6 & $06 / 2019$ & 15 & 25,5 & Sí \\
7 & $02 / 2020$ & 6 & 22 & Sí \\
\hline
\end{tabular}

Mediana (Me). Índice de masa corporal (IMC).

computada de abdomen y pelvis en la evaluación de pacientes post-BPG con dolor abdominal compatible con obstrucción intestinal y que consultan en la urgencia, permite mejorar el diagnóstico en estos pacientes ${ }^{33}$. En cuanto a la técnica de reversión utilizada en los casos de desnutrición, creemos que es preferible usar la técnica de anastomosis gastrogástrica, con el objetivo de poder preservar la mayor cantidad de intestino delgado.

Existen diversas causas potenciales de diarrea crónica post-BPG como lo son el sobrecrecimiento bacteriano, insuficiencia pancreática exocrina, enfermedad inflamatoria intestinal, síndrome de dumping, infección por Clostridium difficile, enfermedad celiaca y alergias alimentarias, entre otras $^{34}$. También podría ser secundaria a aspectos técnicos de la cirugía como, por ejemplo, el largo del asa biliar, alimentaria y común. Por ejemplo, el desarrollo de diarrea crónica es más frecuente en pacientes en los cuales se realizó un BPG distal donde el asa común es más corta comparado con pacientes con BPG standard $^{35}$. En ambos pacientes, la decisión de revertir el bypass se realizó después de haber descartado causas que pudieran ser tratadas de forma específica y tomando en consideración la no respuesta a las intervenciones realizadas y al deterioro en la calidad de vida.

La R-BPG ha demostrado ser una intervención eficaz en el control de las complicaciones descritas. Sin embargo, se debe considerar que es un procedimiento que presenta una morbilidad mayor a la observada en la cirugía bariátrica primaria. Como se puede observar en la Tabla 3, las distintas series reportan complicaciones Clavien-Dindo $\geq$ III entre $0-32 \%$, demostrando la mayor morbilidad que este procedimiento puede llegar a alcanzar ${ }^{18,19}$. Por este motivo, la reversión del BPG debe ser considerada 


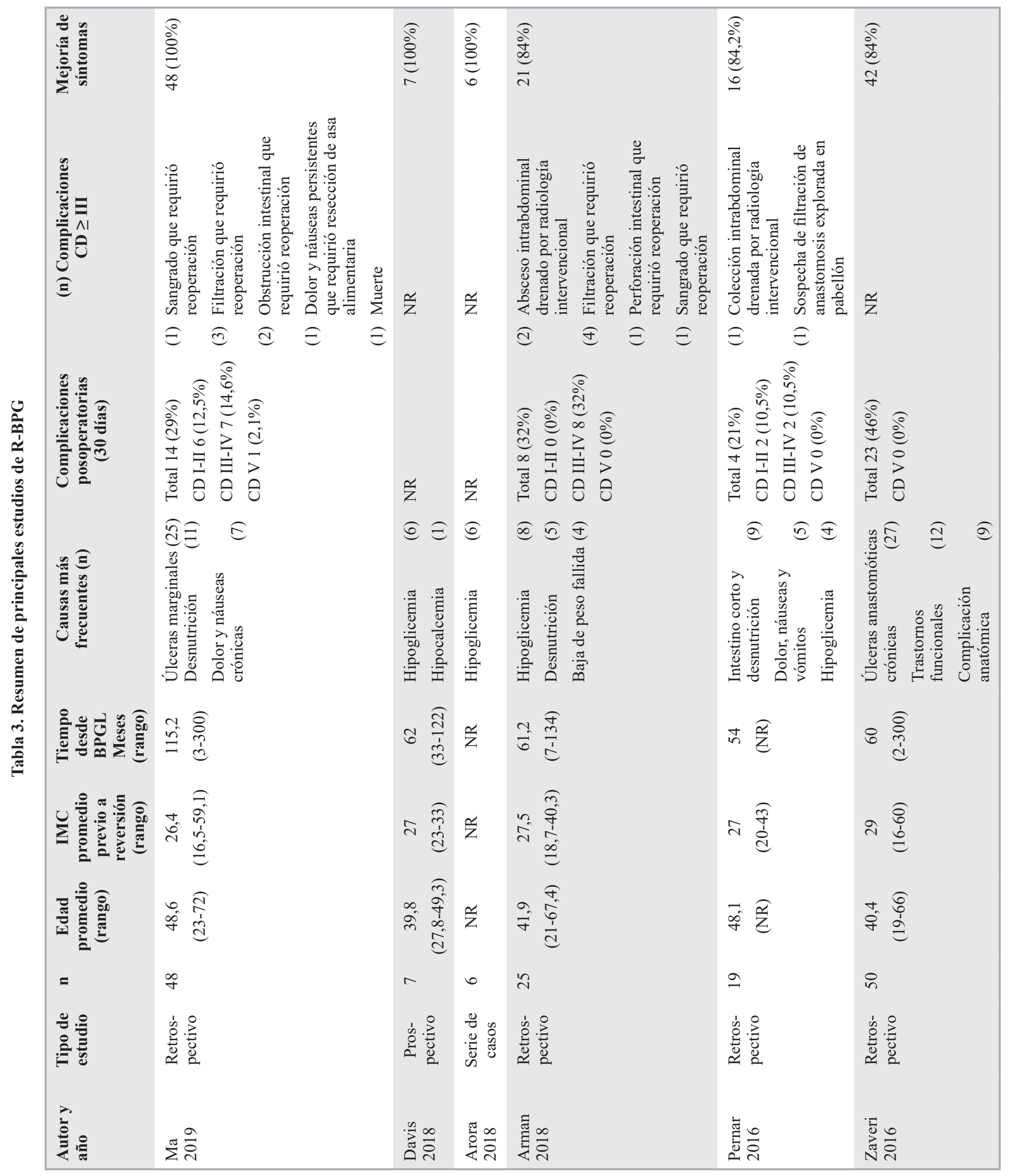




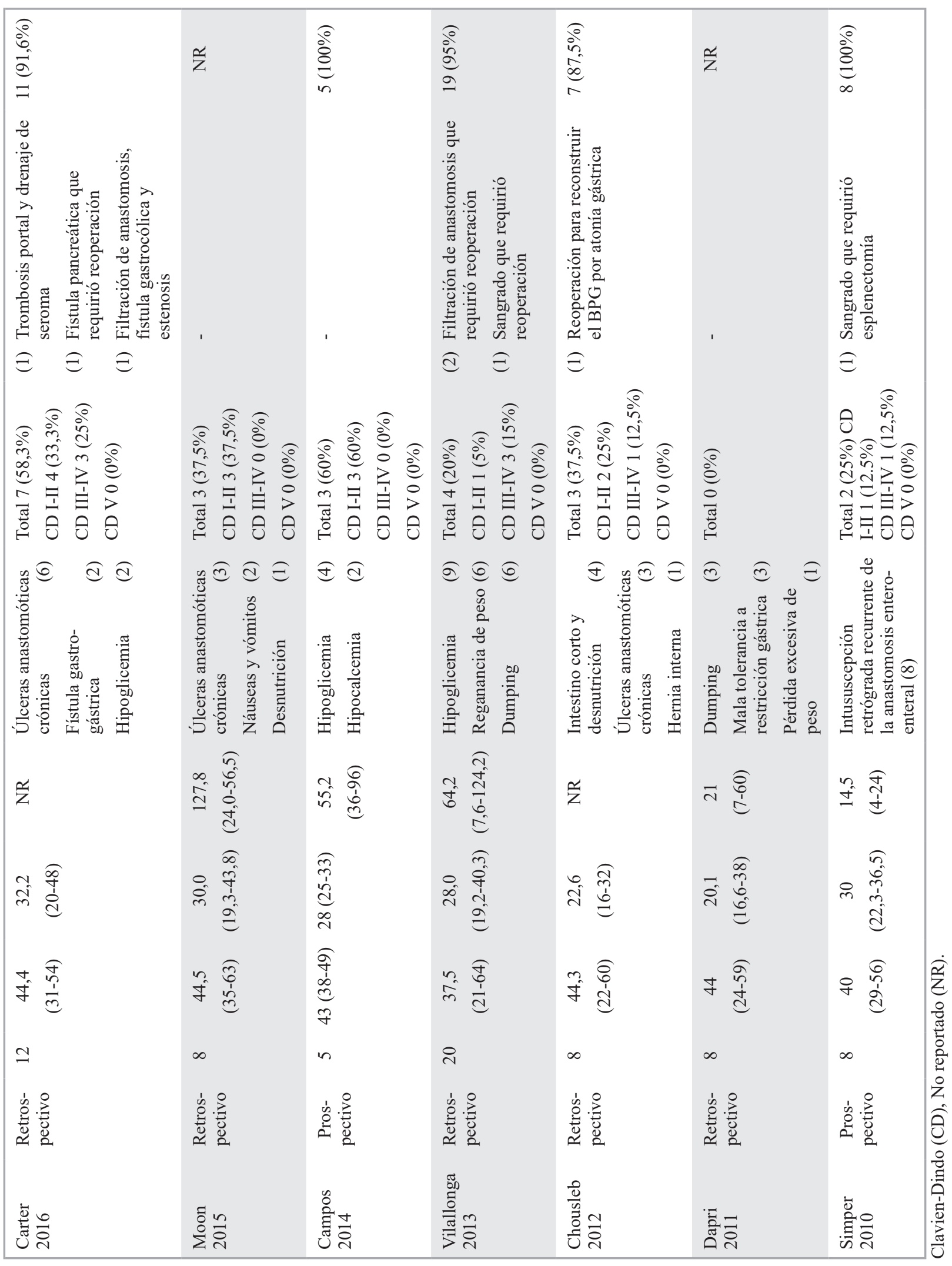


como el último recurso terapéutico y realizada en centros con experiencia. En nuestra serie de casos no tuvimos complicaciones Clavien-Dindo $\geq$ III, lo que coincide con lo reportado por algunos grupos ${ }^{19,23}$. Otros riesgos que existen después de la R-BPG son la recuperación del peso perdido y la falta de reversión completa de la complicación que motivó la cirugía inicial. Para evitar o disminuir la recuperación del peso, algunos grupos consideran la realización de una GM al momento de la reversión ${ }^{16,18}$. Sin embargo, esta debería evitarse cuando la indicación de la reversión es por diarrea crónica o dumping, debido a que el vaciamiento gástrico acelerado descrito después de la GM podría reducir el control de la diarrea o dumping ${ }^{36}$. En nuestra institución priorizamos la resolución de los síntomas que originaron la reversión y consideramos que agregar una intervención adicional no enfocada en la resolución de la complicación podría agregar un riesgo innecesario.

Nuestro estudio cuenta con limitaciones, principalmente aquellas inherentes a su carácter retrospectivo, número reducido de pacientes y seguimiento limitado. Con respecto a las fortalezas, este trabajo es la primera serie de casos reportada en nuestro país de R-BPG, ya que un estudio previo reportaba solo un caso $^{37}$, además se presentaron 2 casos en un Congreso Chileno e Internacional de Cirugía ${ }^{38,39}$.

\section{Conclusiones}

La restauración de la continuidad del tracto gastrointestinal por medio de la R-BPG es una intervención eficaz para controlar complicaciones metabólicas y nutricionales experimentadas en un reducido grupo de pacientes. Debido al mayor riesgo de complicaciones posoperatorias, esta intervención debe ser reservada como último recurso terapeútico, en pacientes evaluados por un equipo multidisiciplinario, en los cuales el manejo médico no logró controlar las complicaciones de la primera intervención.

\section{Responsabilidades éticas}

Protección de personas y animales. Los autores declaran que para esta investigación no se han realizado experimentos en seres humanos ni en animales.

Confidencialidad de los datos. Los autores declaran que en este artículo no aparecen datos de pacientes.

Conflictos de interés: no hay.

\section{Bibliografía}

1. Adams TD, Davidson LE, Hunt SC. Weight and Metabolic Outcomes 12 Years after Gastric Bypass. N Engl J Med. 2018;378:93-6.

2. Sjostrom L, Narbro K, Sjostrom CD, Karason K, Larsson B, Wedel H, et al. Effects of bariatric surgery on mortality in Swedish obese subjects. N Engl J Med. 2007;357:741-52.

3. Ricci C, Gaeta M, Rausa E, Macchitella Y, Bonavina L. Early impact of bariatric surgery on type II diabetes, hypertension, and hyperlipidemia: a systematic review, meta-analysis and meta-regression on 6,587 patients. Obes Surg. 2014;24:522-8.

4. Schauer PR, Bhatt DL, Kirwan JP, Wolski $\mathrm{K}$, Aminian A, Brethauer SA, et al. Bariatric Surgery versus Intensive Medical Therapy for Diabetes - 5-Year Outcomes. N Engl J Med. 2017;376:641-51.

5. Adams TD, Davidson LE, Litwin SE, Kim J, Kolotkin RL, Nanjee MN, et al. Weight and Metabolic Outcomes 12 Years after Gastric Bypass. N Engl J Med. 2017;377:1143-55.

6. Angrisani L, Santonicola A, Iovino P, Formisano G, Buchwald H, Scopinaro N. Bariatric Surgery Worldwide 2013. Obes Surg. 2015;25:1822-32.

7. Himpens J, Dapri G, Cadière GD. Laparoscopic conversion of the gastric bypass into a normal anatomy. Obes Surg. 2006;16:908-12.

8. Brethauer SA, Kothari S, Sudan R, Williams B, English WJ, Brengman M, et al. Systematic review on reoperative bariatric surgery: American Society for Metabolic and Bariatric Surgery Revision Task Force. Surg Obes Relat Dis. 2014;10:952-72.

9. Moon RC, Frommelt A, Teixeira AF, Jawad MA. Indications and outcomes of reversal of Roux-en-Y gastric bypass. Surg Obes Relat Dis. 2015;11:821-6.

10. Pernar LI, Kim JJ, Shikora SA. Gastric bypass reversal: a 7-year experience. Surg Obes Relat Dis. 2016;12:1492-8.

11. Zaveri H, Dallal RM, Cottam D,
Surve A, Kartiko S, Bonnani F, et al. Indications and Operative Outcomes of Gastric Bypass Reversal. Obes Surg. 2016;26:2285-90.

12. Guzman S, Manrique M, Raddatz A, Norero E, Salinas J, Achurra P, et al. Results of bariatric surgery. Experience over 18 years. Rev Med Chile 2013;141:553-61.

13. Clavien PA, Barkun J, de Oliveira ML, Vauthey JN, Dindo D, Schulick RD, et al. The Clavien-Dindo classification of surgical complications: five-year experience. Ann Surg. 2009;250:187-96.

14. Aminian A, Zajichek A, Arterburn DE, Wolski KE, Brethauer SA, Schauer PR, et al. Association of Metabolic Surgery With Major Adverse Cardiovascular Outcomes in Patients With Type 2 Diabetes and Obesity. JAMA 2019;322:1271-1282. doi: 10.1001/jama.2019.14231. Online ahead of print.

15. Ma P, Ghiassi S, Lloyd A, Haddad A, Boone K, DeMaria E, et al. Reversal of Roux en Y gastric bypass: largest single 
institution experience. Surg Obes Relat Dis. 2019;15:1311-6.

16. Carter CO, Fernández AZ, McNatt SS, Powell MS. Conversion from gastric bypass to sleeve gastrectomy for complications of gastric bypass. Surg Obes Relat Dis. 2016;12:572-6.

17. Chousleb E, Patel S, Szomstein S, Rosenthal R. Reasons and operative outcomes after reversal of gastric bypass and jejunoileal bypass. Obes Surg. 2012;22:1611-6.

18. Arman GA, Himpens J, Bolckmans $\mathrm{R}$, Van Compernolle D, Vilallonga R, Leman G. Medium-Term Outcomes after Reversal of Roux-en-Y Gastric Bypass. Obes Surg. 2018;28:781-90.

19. Dapri G, Cadiere GB, Himpens J. Laparoscopic reconversion of Rouxen-Y gastric bypass to original anatomy: technique and preliminary outcomes. Obes Surg. 2011;21:1289-95.

20. Davis DB, Khoraki J, Ziemelis M, Sirinvaravong S, Han JY, Campos GM. Roux en Y gastric bypass hypoglycemia resolves with gastric feeding or reversal: Confirming a non-pancreatic etiology. Mol Metab. 2018;9:15-27.

21. Arora I, Patti ME. Can reversal of RYGB also reverse hypoglycemia? Mol Metab. 2018;9:1-3.

22. Vilallonga R, van de Vrande S, Himpens J. Laparoscopic reversal of Roux-en-Y gastric bypass into normal anatomy with or without sleeve gastrectomy. Surg Endosc. 2013;27:4640-8.

23. Campos GM, Ziemelis M, Paparodis R, Ahmed M, Davis DB. Laparoscopic reversal of Roux-en-Y gastric bypass: technique and utility for treatment of endocrine complications. Surg Obes Relat Dis. 2014;10:36-43.

24. Simper SC, Erzinger JM, McKinlay $\mathrm{RD}$, Smith SC. Laparoscopic reversal of gastric bypass with sleeve gastrectomy for treatment of recurrent retrograde intussusception and Roux stasis syndrome.
Surg Obes Relat Dis. 2010;6:684-8.

25. Pigeyre M, Vaurs C, Raverdy V, Hanaire H, Ritz P, Pattou F. Increased risk of OGTT-induced hypoglycemia after gastric bypass in severely obese patients with normal glucose tolerance. Surg Obes Relat Dis. 2015;11:573-7.

26. Lee CJ, Clark JM, Schweitzer M, Magnuson T, Steele K, Koerner O, et al. Prevalence of and risk factors for hypoglycemic symptoms after gastric bypass and sleeve gastrectomy. Obesity (Silver Spring). 2015;23:1079-84.

27. Kefurt R, Langer FB, Schindler K, Shakeri-Leidenmuhler S, Ludvik B, Prager G. Hypoglycemia after RouxEn-Y gastric bypass: detection rates of continuous glucose monitoring (CGM) versus mixed meal test. Surg Obes Relat Dis. 2015;11:564-9.

28. Rao BB, Click B, Eid G, Codario RA. Management of Refractory Noninsulinoma Pancreatogenous Hypoglycemia Syndrome with Gastric Bypass Reversal: A Case Report and Review of the Literature. Case Rep Endocrinol. 2015;2015:384526.

29. Mala T. Postprandial hyperinsulinemic hypoglycemia after gastric bypass surgical treatment. Surg Obes Relat Dis. 2014;10:1220-5.

30. Rodríguez A, Mosti M, Sierra M, PérezJohnson R, Flores S, Domínguez G, et al. Small bowel obstruction after antecolic and antegastric laparoscopic Roux-en-Y gastric bypass: could the incidence be reduced? Obes Surg. 2010;20:1380-4.

31. Koppman JS, Li C, Gandsas A. Small bowel obstruction after laparoscopic Roux-en-Y gastric bypass: a review of 9,527 patients. J Am Coll Surg. 2008;206:571-84.

32. Stenberg E, Szabo E, Agren G, Ottosson J, Marsk R, Lonroth H, et al. Closure of mesenteric defects in laparoscopic gastric bypass: a multicentre, randomised, parallel, open-label trial. Lancet
2016;387(10026):1397-404.

33. Frokjaer JB, Jensen WN, Holt G, Omar HK, Olesen SS. The diagnostic performance and interrater agreement of seven CT findings in the diagnosis of internal hernia after gastric bypass operation. Abdom Radiol (NY). 2018;43:3220-6.

34. Sollier C, Barsamian C, Bretault M, Poghosyan T, Rahmi G, Chevallier JM, et al. Diagnostic and Therapeutic Management of Post-Gastric Bypass Chronic Diarrhea: a Systematic Review. Obes Surg. 2020;30:1102-11.

35. Risstad H, Svanevik M, Kristinsson JA, Hjelmesaeth J, Aasheim ET, Hofso D, et al. Standard vs Distal Roux-en-Y Gastric Bypass in Patients With Body Mass Index 50 to 60: A Double-blind, Randomized Clinical Trial. JAMA Surg. 2016;151:1146-55.

36. Li M, Liu Y, Jin L, Wang W, Zeng $\mathrm{N}$, Wang $\mathrm{L}$, et al. Alterations of Gastric Emptying Features Following Laparoscopic Sleeve Gastrectomy in Chinese Patients with Obesity: a SelfControlled Observational Study. Obes Surg. 2019;29:617-25.

37. Caris G, Almarza R, Canales C, Sabra D. Reversión laparoscópica de bypass gástrico con Y de Roux a anatomía normal, en paciente con malnutrición severa y diarrea: reporte de un caso. Rev Chil Cir. 2017;69:162-6.

38. Saba J, Bravo M, Zajjur J, Pérez A, Rivas E, Fernández R. Reaparición de la diabetes tipo 2 luego de revertir el bypass gástrico. Reporte de caso. $89^{\circ}$ Congreso Chileno e Internacional de Cirugía; 2016 nov 27-30; La Serena, Chile.

39. Bravo D, Villao M, Moya R, Bravo E, Bustamante M. Úlcera marginal perforada post Bypass Gástrico (BG). Manejo con laparotomía exploradora para control de daños y reversión de $\mathrm{BG}$ posterior. $90^{\circ}$ Congreso Chileno e Internacional de Cirugía; 2017 nov 1-4; Pucón, Chile. 